\title{
Upper semicontinuity of uniform attractors for nonclassical diffusion equations
}

Yonghai Wang ${ }^{*}$, Pengrui Li and Yuming Qin

"Correspondence:

yonghai@dhu.edu.cn

Department of Applied

Mathematics, Donghua University,

Shanghai, 201620, P.R. China

\begin{abstract}
We study the upper semicontinuity of a uniform attractor for a nonautonomous nonclassical diffusion equation with critical nonlinearity. In particular, we prove that the uniform (with respect to (w.r.t.) $g \in \Sigma$ ) attractor $\mathcal{A}_{\Sigma}^{\varepsilon}(\varepsilon \geq 0)$ for equation (1.1) satisfies $\lim _{\varepsilon \rightarrow \varepsilon_{0}} \operatorname{dist}_{H_{0}^{1}(\Omega)}\left(\mathcal{A}_{\Sigma}^{\varepsilon}, \mathcal{A}_{\Sigma}^{\varepsilon_{0}}\right)=0$ for any $\varepsilon_{0} \geq 0$.
\end{abstract}

MSC: $37 \mathrm{~L} 05 ; 35 B 40 ; 35 B 41$

Keywords: nonautonomous; nonclassical diffusion equation; uniform attractor; upper semicontinuity

\section{Introduction}

This paper is devoted to studying the following nonautonomous nonclassical diffusion equation:

$$
\left\{\begin{array}{l}
\partial_{t} u-\varepsilon \Delta \partial_{t} u-\Delta u+f(u)=g(x, t), \quad \text { in } \Omega \times(\tau, \infty), \\
u(x, \tau)=u_{\tau},\left.\quad u(x, t)\right|_{\partial \Omega \times[\tau, \infty)}=0,
\end{array}\right.
$$

in a bounded domain $\Omega \subset \mathbb{R}^{N}(N \geq 3)$ with smooth boundary $\partial \Omega$ and perturbed parameter $\varepsilon \geq 0$.

For the nonlinearity $f \in C^{1}(\mathbb{R})$, we assume it satisfies the following growth and dissipation conditions:

$$
\begin{gathered}
f^{\prime}(s) \leq C\left(1+|s|^{\frac{4}{N-2}}\right), \\
\liminf _{|u| \rightarrow \infty} f^{\prime}(u)>-\lambda_{1},
\end{gathered}
$$

where $C>0$ and $\lambda_{1}$ is the first eigenvalue of $-\Delta$ on $H_{0}^{1}(\Omega)$.

For the external force $g(x, t)$, we assume

$$
g(x, t), \quad \partial_{t} g(x, t) \in L_{b}^{2}\left(\mathbb{R} ; L^{2}(\Omega)\right)
$$

where the translation bounded space $L_{b}^{2}\left(\mathbb{R} ; L^{2}(\Omega)\right)$ is defined by the following norm:

$$
\|g(x, t)\|_{L_{b}^{2}}:=\sup _{t \in \mathbb{R}}\|g(x, t)\|_{L^{2}\left(t, t+1 ; L^{2}(\Omega)\right)}^{2}
$$

(c) The Author(s) 2017. This article is distributed under the terms of the Creative Commons Attribution 4.0 International License (http://creativecommons.org/licenses/by/4.0/), which permits unrestricted use, distribution, and reproduction in any medium, provided you give appropriate credit to the original author(s) and the source, provide a link to the Creative Commons license, and indicate if changes were made. 
Nonclassical diffusion equations arise in fluid mechanics, solid mechanics, and the theory of heat conduction; they describe that the diffusing species behave as a linearly viscous fluid (see, e.g., [1, 2]). Asymptotic behavior of equations analogous to (1.1) has been investigated in many literature works during the last years (see, e.g., [3-12] and the references therein).

Since equation (1.1) reduces to a usual reaction-diffusion equation when $\varepsilon=0$, it is natural to examine the limiting behavior of solutions to equation (1.1) when $\varepsilon$ goes to 0 . This problem has been considered by some authors. In [10], the authors study the existence of global attractors in $H^{2}(\Omega) \cap H_{0}^{1}(\Omega)$ (generated by strong solutions), and their upper semicontinuity in $H_{0}^{1}(\Omega)$ for the autonomous case of equation (1.1) (that is, the external force $g$ is independent of $t$ ) with subcritical nonlinearity. In [4], the upper semicontinuity of pullback attractors in $L^{2}(\Omega)$ for equation (1.1) with subcritical nonlinearity was considered. In [13], the upper semicontinuity of global attractors in $H^{1}\left(\mathbb{R}^{n}\right)$ for equation (1.1) defined in unbounded domains with subcritical nonlinearity was considered. As far as we know, there are no results as to the upper semicontinuity of uniform attractors generated by (weak) solutions in $H_{0}^{1}(\Omega)$ for equation (1.1) with critical nonlinearity.

In order to construct uniform attractors for equation (1.1), it is necessary to define a proper symbol space generated by external force $g(x, t)$ (see, e.g., [14]). Let $L_{w, \text { loc }}^{2}\left(\mathbb{R} ; L^{2}(\Omega)\right.$ ) denote the locally square integrable (in time) space $L_{\text {loc }}^{2}\left(\mathbb{R} ; L^{2}(\Omega)\right)$ endowed with the locally weak convergence topology. For every $g_{0} \in L_{b}^{2}\left(\mathbb{R} ; L^{2}(\Omega)\right)$, we define a set of functions obtained by all time shifts of $g_{0}$ as follows:

$$
\Sigma_{0}=\left\{(x, t) \rightarrow g_{0}(x, t+h) \mid h \in \mathbb{R}\right\} .
$$

The hull of $g_{0}$, denoted by $\Sigma=\mathcal{H}\left(g_{0}\right)$, is defined as a closure of $\Sigma_{0}$ in the topology of $L_{w, \text { loc }}^{2}\left(\mathbb{R} ; L^{2}(\Omega)\right)$ (see, e.g., [14], Section V.4.). We choose $\Sigma$ as the symbol space for equation (1.1).

From $[9,15]$, we know that equation $(1.1)$ is globally well-posed in $H_{0}^{1}(\Omega)$ for every $\varepsilon \geq 0$, $g \in \Sigma$, the solution operator $U_{g}^{\varepsilon}(t, \tau)$ forms a process (see (2.1), (2.2)) in $H_{0}^{1}(\Omega)$ and satisfies the following assumptions (translation identity):

$$
\begin{aligned}
& U_{T(s) g}^{\varepsilon}(t, \tau)=U_{g}^{\varepsilon}(t+s, \tau+s), \quad \forall g \in \Sigma, s \geq 0, t \geq \tau, \tau \in \mathbb{R} ; \\
& T(s) \Sigma=\Sigma, \quad \forall s \geq 0,
\end{aligned}
$$

where $\{T(s)\}_{s \geq 0}$ is the translation semigroup on $\Sigma$.

The existence of uniform (with respect to (w.r.t.) $g \in \Sigma$ ) attractors in different kinds of phase spaces for equations analogous to equation (1.1) $(\varepsilon=0$ or $\varepsilon>0)$ with more general nonlinearities and external forces have been investigated in many literature works (see, e.g., $[9,14-18]$ and the references therein). We can summarize the following result (see, e.g., $[8,9,14,15]$ and the references therein).

Theorem 1.1 Let (1.2), (1.3) be satisfied. Assume that $g_{0} \in L_{b}^{2}\left(\mathbb{R} ; L^{2}(\Omega)\right)$ and $\Sigma$ is the hull of $g_{0}$ in $L_{w, \text { loc }}^{2}\left(\mathbb{R} ; L^{2}(\Omega)\right)$. For each $\varepsilon \geq 0$, the family of processes $\left\{U_{g}^{\varepsilon}(t, \tau)\right\}, g \in \Sigma$ associated with equation (1.1) possesses a compact uniform (w.r.t. $g \in \Sigma$ ) attractor $\mathcal{A}_{\Sigma}^{\varepsilon}$ in $H_{0}^{1}(\Omega)$. 
Moreover, this attractor satisfies that

$$
\mathcal{A}_{\Sigma}^{\varepsilon}=\omega_{\tau, \Sigma}^{\varepsilon}(\mathcal{B})=\bigcup_{g \in \Sigma} \mathcal{K}_{g}^{\varepsilon}(s), \quad \forall \tau, s \in \mathbb{R}
$$

where $\mathcal{B}$ is a uniformly (w.r.t. $g \in \Sigma$ ) absorbing set, which is independent of $\varepsilon$ (see Corollary 3.1) and $\omega_{\tau, \Sigma}^{\varepsilon}(\mathcal{B})$ is the $\omega$-limit set of $\mathcal{B}, \mathcal{K}_{g}^{\varepsilon}(s)$ is the kernel section of the process $\left\{U_{g}^{\varepsilon}(t, \tau)\right\}$ at time $t=s$.

The aim of this paper is to obtain the upper semicontinuity of uniform attractors in $H_{0}^{1}(\Omega)$ for equation (1.1), especially the nonlinear term $f$ has a critical exponent (see (1.2)). More precisely, the main result of this paper can be stated as follows, which will be proved in Section 3 later.

Theorem 1.2 Let (1.2), (1.3) be satisfied. Assume $g_{0}, \partial_{t} g_{0} \in L_{b}^{2}\left(\mathbb{R} ; L^{2}(\Omega)\right)$ and $\Sigma$ is the hull of $g_{0}$ in $L_{w, \text { loc }}^{2}\left(\mathbb{R} ; L^{2}(\Omega)\right)$. Let $\mathcal{A}_{\Sigma}^{\varepsilon}(\varepsilon \geq 0)$ be the uniform attractor given by Theorem 1.1, then it satisfies that for every $\varepsilon_{0} \geq 0$,

$$
\lim _{\varepsilon \rightarrow \varepsilon_{0}} \operatorname{dist}_{H_{0}^{1}}\left(A_{\Sigma}^{\varepsilon}, A_{\Sigma}^{\varepsilon_{0}}\right)=0
$$

and

$$
\lim _{\varepsilon \rightarrow \varepsilon_{0}} \operatorname{dist}_{H_{0}^{1}}\left(\mathcal{K}_{g}^{\varepsilon}(s), \mathcal{K}_{g}^{\varepsilon_{0}}(s)\right)=0, \quad \forall g \in \Sigma, \forall s \in \mathbb{R}
$$

where dist $_{H_{0}^{1}}$ denotes the standard Hausdorff semidistance in $H_{0}^{1}(\Omega)$.

Hereafter, we denote by $\langle\cdot, \cdot\rangle$ and $\|\cdot\|$ the inner product and the norm in $L^{2}(\Omega)$, respectively. The symbols $C$ and $Q$ stand for a generic positive constant and a generic positive increasing function, respectively. Young's and Hölder's inequalities will be applied without explicit mention.

\section{Preliminaries}

In this section, we recall some basic concepts and results of the theory of uniform attractors, we refer to $[14,19]$ and the references therein for more details.

Let $X$ be a Banach space and $\Sigma$ be a parameter set. The set of operators $\left\{U_{g}(t, \tau)\right\}, g \in \Sigma$ is called a family of evolution processes in $X$ with symbol space $\Sigma$ if, for any $g \in \Sigma$, it satisfies

$$
\begin{aligned}
& U_{g}(t, \tau)=U_{g}(t, s) U_{g}(s, \tau), \quad \forall t \geq s \geq \tau, \tau \in \mathbb{R} ; \\
& U_{g}(\tau, \tau)=\text { Id (Identity), } \quad \forall \tau \in \mathbb{R} .
\end{aligned}
$$

Definition 2.1 (see [14]) A bounded subset $\mathcal{B}$ of $X$ is said to be uniformly (w.r.t. $g \in \Sigma$ ) absorbing for the family of processes $\left\{U_{g}(t, \tau)\right\}, g \in \Sigma$ if, for any $\tau \in \mathbb{R}$ and any bounded subset $B \subset X$, there exists $T=T(B) \geq \tau$ such that $\bigcup_{t \geq T} \bigcup_{g \in \Sigma} U_{g}(t, \tau) B \subset \mathcal{B}$.

Definition 2.2 (see [14]) A closed set $\mathcal{A}_{\Sigma}$ of $X$ is said to be a uniform (w.r.t. $g \in \Sigma$ ) attractor of the family of processes $\left\{U_{g}(t, \tau)\right\}, g \in \Sigma$ if 
(i) $\mathcal{A}_{\Sigma}$ is uniformly (w.r.t. $g \in \Sigma$ ) attracting, that is, for any $\tau \in \mathbb{R}$ and any bounded subset $B \subset X, \lim _{t \rightarrow \infty} \sup _{g \in \Sigma} \operatorname{dist}_{X}\left(U_{g}(t, \tau) B, \mathcal{B}\right)=0$ (attracting property);

(ii) $\mathcal{A}_{\Sigma}$ is contained in any closed uniformly attracting set (minimality property).

Definition 2.3 (see [20]) A family of processes $\left\{U_{g}(t, \tau)\right\}, g \in \Sigma$ on $X$ is said to be uniformly (w.r.t. $g \in \Sigma$ ) asymptotically compact if and only if, for any $\tau \in \mathbb{R},\left\{g_{n}\right\}_{n \in \mathbb{N}} \subset \Sigma$, $\left\{t_{n}\right\}_{n \in \mathbb{N}} \subset[\tau, \infty)$ with $t_{n} \rightarrow \infty(n \rightarrow \infty)$ and any bounded sequence $\left\{x_{n}\right\}_{n \in \mathbb{N}} \subset X$, the sequence $\left\{U_{g_{n}}\left(t_{n}, \tau\right) x_{n}\right\}_{n \in \mathbb{N}}$ is relatively compact in $X$.

Definition 2.4 (see [14]) For any bounded subset $B$ of $X$, the uniform (w.r.t. $g \in \Sigma$ ) $\omega$-limit set $\omega_{\tau, \Sigma}(B)$ for the family of processes $\left\{U_{g}(t, \tau)\right\}, g \in \Sigma$ is defined by

$$
\omega_{\tau, \Sigma}(B)=\bigcap_{t \geq \tau} \bar{\bigcup}_{g \in \Sigma} U_{g}(s, \tau) B
$$

Assumption I Let $\{T(s)\}_{s \geq 0}$ be a family of operators acting on $\Sigma$ and satisfying

(i) $T(s) \Sigma=\Sigma, \forall s \geq 0$;

(ii) translation identity

$$
U_{g}(t+s, \tau+s)=U_{T(s) g}(t, \tau), \quad \forall g \in \Sigma, t \geq \tau, \tau \in \mathbb{R}, s \geq 0 .
$$

We recall (see, e.g., [14]) that the kernel $\mathcal{K}$ of the process $\{U(t, \tau)\}$ acting on $X$ consists of all bounded complete trajectories of $\{U(t, \tau)\}$, i.e.,

$$
\mathcal{K}=\left\{u(\cdot) \mid\|u(t)\|_{X} \leq C_{u}, U(t, \tau) u(\tau)=u(t), \forall t \geq \tau, \tau \in \mathbb{R}\right\}
$$

and $\mathcal{K}(s)=\{u(s) \mid u(\cdot) \in \mathcal{K}\}$ is said to be the kernel section at time $t=s, s \in \mathbb{R}$.

Definition 2.5 A family of processes $\left\{U_{\sigma}(t, \tau)\right\}, g \in \Sigma$ is said to be $(X \times \Sigma, X)$-weakly continuous if, for arbitrary fixed $t \geq \tau, \tau \in \mathbb{R}$, the mapping $(u, g) \rightarrow U_{g}(t, \tau) u$ is weakly continuous from $X \times \Sigma$ to $X$.

Assumption II Let $\Sigma$ be a weakly compact set and $\left\{U_{g}(t, \tau)\right\}, g \in \Sigma$ be $(X \times \Sigma, X)$-weakly continuous.

Theorem 2.1 (see [21, 22]) Under Assumptions I, II, if $\left\{U_{g}(t, \tau)\right\}, g \in \Sigma$

(i) has a uniformly (w.r.t. $g \in \Sigma$ ) absorbing set $\mathcal{B}$;

(ii) is uniformly (w.r.t. $g \in \Sigma$ ) asymptotically compact,

then $\left\{U_{g}(t, \tau)\right\}, g \in \Sigma$ has a compact uniform (w.r.t. $g \in \Sigma$ ) attractor $\mathcal{A}_{\Sigma}$ satisfying

$$
\mathcal{A}_{\Sigma}=\omega_{\tau, \Sigma}(\mathcal{B})=\bigcup_{g \in \Sigma} \mathcal{K}_{g}(s), \quad \forall \tau, s \in \mathbb{R}
$$

where $\mathcal{K}_{g}$ is the kernel section of the process $U_{g}(t, \tau)$ and $\mathcal{K}_{g}(s)$ is the kernel section at $t=s$. 


\section{Upper semicontinuity of uniform attractors}

The following result about the existence and uniqueness of solutions of equation (1.1) can be obtained by the standard Faedo-Galerkin methods, here we only formulate the result.

Theorem 3.1 Let (1.2), (1.3) be satisfied and $g(x, t) \in L_{\text {loc }}^{2}\left(\mathbb{R} ; L^{2}(\Omega)\right)$. For any $\varepsilon \geq 0, I=$ $[\tau, T]$ and $u_{\tau} \in H_{0}^{1}(\Omega)$, equation (1.1) admits a unique solution $u$ satisfying

$$
u \in C\left(I ; H_{0}^{1}(\Omega)\right), \quad \partial_{t} u \in L^{2}\left(I ; H_{0}^{1}(\Omega)\right) .
$$

Moreover, the solution continuously depends on the initial data in $H_{0}^{1}(\Omega)$.

By Theorem 3.1, for each $\varepsilon \geq 0$ and $g \in L_{b}^{2}\left(\mathbb{R} ; L^{2}(\Omega)\right)$, we define a process as follows:

$$
U_{g}^{\varepsilon}(t, \tau) u_{\tau}=u(t) \quad \text { for all } t \geq \tau \text { and } u_{\tau} \in H_{0}^{1}(\Omega)
$$

and the mapping $U_{g}^{\varepsilon}(t, \tau): H_{0}^{1}(\Omega) \rightarrow H_{0}^{1}(\Omega)$ is continuous.

Lemma 3.1 Let (1.2), (1.3) be satisfied. Assume $g_{0} \in L_{b}^{2}\left(\mathbb{R} ; L^{2}(\Omega)\right)$ and $\Sigma$ is the hull of $g_{0}$ in $L_{w, \text { loc }}^{2}\left(\mathbb{R} ; L^{2}(\Omega)\right)$. There exists $\delta>0$ such that for any $\tau \in \mathbb{R}$ and any initial data $u_{\tau} \in H_{0}^{1}(\Omega)$, the solutions of equation (1.1) satisfy: for all $\varepsilon \geq 0, t \geq \tau$ and $g \in \Sigma$,

$$
\begin{aligned}
& \|\nabla u(t)\|^{2} \leq e^{-\delta(t-\tau)} Q\left(\left\|\nabla u_{\tau}\right\|\right)+M_{0}, \\
& \int_{t}^{t+1}\left(\left\|\partial_{t} u(s)\right\|^{2}+\varepsilon\left\|\nabla \partial_{t} u(s)\right\|^{2}\right) d s \leq Q\left(\left\|\nabla u_{\tau}\right\|\right)+M_{0},
\end{aligned}
$$

where $M_{0}>0$ depends on $\left\|g_{0}\right\|_{L_{b}^{2}}$, but is independent of $\varepsilon$.

Proof The proof is classical (see, e.g., [8-10]), we only sketch the main steps of the reasoning. Multiplying the first equation of (1.1) by $\partial_{t} u+\delta u$ and integrating over $\Omega$, we have

$$
\begin{aligned}
& \frac{d}{d t}\left(\delta\|u\|^{2}+(1+\varepsilon \delta)\|\nabla u\|^{2}+2\langle F(u), 1\rangle\right)+2\left\|\partial_{t} u\right\|^{2}+2 \varepsilon\left\|\nabla \partial_{t} u\right\|^{2} \\
& \quad+2 \delta\|\nabla u\|^{2}+2 \delta\langle f(u), u\rangle=2\left\langle g(x, t), \partial_{t} u+\delta u\right\rangle,
\end{aligned}
$$

where $F(u)=\int_{0}^{u} f(s) d s$ and $\delta>0$ is sufficiently small which will be given precisely later.

Observe that

$$
2\left\langle g(x, t), \partial_{t} u+\delta u\right\rangle \leq 2\|g(x, t)\|^{2}+\left\|\partial_{t} u\right\|^{2}+\delta^{2}\|u\|^{2} .
$$

Thus

$$
\frac{d}{d t} E(t)+\delta E(t)+\left\|\partial_{t} u\right\|^{2}+2 \varepsilon\left\|\nabla \partial_{t} u\right\|^{2}+\Pi(t) \leq 2\|g(x, t)\|^{2},
$$

where

$$
E(t)=\delta\|u\|^{2}+(1+\varepsilon \delta)\|\nabla u\|^{2}+2\langle F(u), 1\rangle+2 C_{\rho}
$$


and

$$
\Pi(t)=2 \delta\|\nabla u\|^{2}+2 \delta\langle f(u), u\rangle-\delta^{2}\|u\|^{2}-\delta E(t) .
$$

Thanks to (1.2) and (1.3), we estimate that

$$
\begin{aligned}
& \langle f(u), u\rangle \geq-\rho\|u\|^{2}-C_{\rho}, \\
& \langle F(u), 1\rangle \geq-\frac{1}{2} \rho\|u\|^{2}-C_{\rho}, \\
& \langle f(u), u\rangle-\langle F(u), 1\rangle \geq-\frac{1}{2} \rho\|u\|^{2}-C_{\rho}, \\
& \langle F(u), 1\rangle \leq C\left(\|\nabla u\|^{\frac{2 N}{N-2}}+1\right)
\end{aligned}
$$

for some positive constants $\rho<\lambda_{1}$ and $C_{\rho}$.

$$
\begin{aligned}
& \text { Let } 0<\delta \leq \frac{1-\rho \lambda_{1}^{-1}}{1+2 \lambda_{1}^{-1}} \text {, then } \\
& E(t) \geq 0 \text { and } \Pi(t) \geq-C_{0},
\end{aligned}
$$

where $C_{0}=4 \delta C_{\rho}$. Moreover, from (3.7), (3.9), there exist positive constants $C_{1}, C_{2}$ such that

$$
C_{1}\|\nabla u\|^{2} \leq E(t) \leq C_{2}\left(\|\nabla u\|^{\frac{2 N}{N-2}}+1\right)
$$

Hence, by (3.5) and (3.10), we get

$$
\frac{d}{d t} E(t)+\delta E(t)+\left\|\partial_{t} u\right\|^{2}+2 \varepsilon\left\|\nabla \partial_{t} u\right\|^{2} \leq 2\|g(x, t)\|^{2}+C_{0} .
$$

Note that [14], Proposition V.4.2, implies that $\|g\|_{L_{b}^{2}} \leq\left\|g_{0}\right\|_{L_{b}^{2}}, \forall g \in \Sigma$. Applying Gronwall's inequality to (3.12), we obtain

$$
E(t) \leq e^{-\delta(t-\tau)} E(\tau)+C\left(\left\|g_{0}\right\|_{L_{b}^{2}}^{2}+1\right), \quad \forall g \in \Sigma,
$$

and this together with (3.11) implies (3.1).

Finally, integrating (3.12) over $[t, t+1]$ with $\delta=0$ leads to (3.2).

By Lemma 3.1, we can construct a uniformly (w.r.t. $g \in \Sigma, \varepsilon \in[0, \infty)$ ) absorbing set, which is independent of $\varepsilon$, for the family of processes $\left\{U_{g}^{\varepsilon}(t, \tau) \mid g \in \Sigma, \varepsilon \in[0, \infty)\right\}$.

Corollary 3.1 Under the assumptions of Lemma 3.1, there exists a bounded uniformly (w.r.t. $g \in \Sigma$ and $\varepsilon \in[0, \infty)$ ) absorbing set $\mathcal{B}$ of $H_{0}^{1}(\Omega)$ for the family of processes $\left\{U_{g}^{\varepsilon}(t, \tau) \mid\right.$ $g \in \Sigma, \varepsilon \in[0, \infty)\}$ associated with equation (1.1), that is, for any $\tau \in \mathbb{R}$ and any bounded subset $B \subset H_{0}^{1}(\Omega)$, there exists $T=T(B) \geq \tau$ such that $\bigcup_{\varepsilon \geq 0} \bigcup_{g \in \Sigma} U_{g}^{\varepsilon}(t, \tau) B \subset \mathcal{B}$ for all $t \geq T$. 
Lemma 3.2 Let (1.2), (1.3) be satisfied. Assume $g_{0}, \partial_{t} g_{0} \in L_{b}^{2}\left(\mathbb{R} ; L^{2}(\Omega)\right)$ and $\Sigma$ is the hull of $g_{0}$ in $L_{w, \mathrm{loc}}^{2}\left(\mathbb{R} ; L^{2}(\Omega)\right)$. For any $\tau \in \mathbb{R}, T>\tau$, any initial data $u_{\tau} \in H_{0}^{1}(\Omega)$ and any $g \in \Sigma$, the solutions of equation (1.1) satisfy the following estimate:

$$
\left\|\partial_{t} u(t)\right\|^{2}+\varepsilon\left\|\nabla \partial_{t} u(t)\right\|^{2} \leq \frac{Q}{(t-\tau)^{2}}, \quad \forall t \in(\tau, T], \forall \varepsilon \geq 0,
$$

where $Q$ depends on $\tau, T,\left\|\nabla u_{\tau}\right\|,\left\|g_{0}\right\|_{L_{b}^{2}}$ and $\left\|\partial_{t} g_{0}\right\|_{L_{b}^{2}}$, but is independent of $\varepsilon$.

Proof From Lemma 3.1, we observe that

$$
\int_{\tau}^{T}\left(\left\|\partial_{t} u(t)\right\|^{2}+\varepsilon\left\|\nabla \partial_{t} u(t)\right\|^{2}\right) d t \leq M,
$$

where $M$ depends on $\tau, T,\left\|\nabla u_{\tau}\right\|$ and $\left\|g_{0}\right\|_{L_{b}^{2}}$.

Differentiate the first equation of (1.1) with respect to $t$ and let $v=\partial_{t} u$, then $v$ satisfies the following equality:

$$
\partial_{t} v-\varepsilon \Delta \partial_{t} v-\Delta v+f^{\prime}(u) v=\partial_{t} g(x, t) .
$$

Multiplying (3.15) by $v$ and integrating over $\Omega$, using (1.3), after standard transformations, we obtain

$$
\frac{d}{d t} G_{\varepsilon}(t)+\|\nabla v\|^{2} \leq 2 l G_{\varepsilon}(t)+\lambda_{1}^{-1}\left\|\partial_{t} g(x, t)\right\|^{2}
$$

for some $l \geq \lambda_{1}$, where $G_{\varepsilon}(t)=\|v(t)\|^{2}+\varepsilon\|\nabla v(t)\|^{2}$.

Multiplying (3.16) by $(t-\tau)^{2}$, we obtain

$$
\begin{aligned}
& \frac{d}{d t}(t-\tau)^{2} G_{\varepsilon}(t) \\
& \quad \leq 2 l(t-\tau)^{2} G_{\varepsilon}(t)+2(t-\tau) G_{\varepsilon}(t)+\lambda_{1}^{-1}(t-\tau)^{2}\left\|\partial_{t} g(x, t)\right\|^{2} \\
& \quad \leq(2 l+1)(t-\tau)^{2} G_{\varepsilon}(t)+G_{\varepsilon}(t)+\lambda_{1}^{-1}(t-\tau)^{2}\left\|\partial_{t} g(x, t)\right\|^{2} .
\end{aligned}
$$

Then, by Gronwall's inequality, [14], Proposition V.4.2, (3.14) and noting that

$$
\int_{\tau}^{T}\left\|\partial_{t} g(x, s)\right\|^{2} d s \leq([T-\tau]+1)\left\|\partial_{t} g\right\|_{L_{b}^{2}}^{2} \leq([T-\tau]+1)\left\|\partial_{t} g_{0}\right\|_{L_{b}^{2}}^{2},
$$

we obtain (3.13) immediately.

Lemma 3.3 Under the assumptions of Lemma 3.2, for any $\tau \in \mathbb{R}$, any bounded subsets $B \subset H_{0}^{1}(\Omega)$ and $I \subset[0, \infty)$, the following estimate holds true:

$$
\begin{aligned}
& \sup _{g \in \Sigma}\left\|\nabla\left(U_{g}^{\varepsilon_{1}}(t, \tau) u_{1}-U_{g}^{\varepsilon_{2}}(t, \tau) u_{2}\right)\right\|^{2} \leq Q\left(\left\|\nabla\left(u_{1}-u_{2}\right)\right\|^{2}+\left|\varepsilon_{1}-\varepsilon_{2}\right|\right), \\
& \forall t \geq \tau, \forall u_{1}, u_{2} \in B, \forall \varepsilon_{1}, \varepsilon_{2} \in I,
\end{aligned}
$$

where $Q$ depends on $t, \tau,\left\|g_{0}\right\|_{L_{b}^{2}},\left\|\partial_{t} g_{0}\right\|_{L_{b}^{2}},|I|$ and $H_{0}^{1}$-bounds of $B$. 
Proof Let $u_{i}(t)=U_{g}^{\varepsilon_{i}}(t, \tau) u_{i}$ be the solution of problem (1.1) with $\varepsilon=\varepsilon_{i}$ and the initial data $u_{i}(\tau)=u_{i}(i=1,2)$.

Set $w(t)=u_{1}(t)-u_{2}(t)$, then the following equality holds true:

$$
\partial_{t} w-\Delta w-\varepsilon_{2} \Delta \partial_{t} w-\left(\varepsilon_{1}-\varepsilon_{2}\right) \Delta \partial_{t} u_{1}+f\left(u_{1}\right)-f\left(u_{2}\right)=0,
$$

with the initial data $w(\tau)=u_{1}-u_{2}$.

Multiplying equation (3.17) by $w$ and integrating over $\Omega$, gives

$$
\begin{aligned}
& \frac{d}{d t}\left(\|w\|^{2}+\varepsilon_{2}\|\nabla w\|^{2}\right)+2\|\nabla w\|^{2} \\
& \quad+2\left(\varepsilon_{1}-\varepsilon_{2}\right)\left\langle-\Delta \partial_{t} u_{1}, w\right\rangle+2\left\langle f\left(u_{1}\right)-f\left(u_{2}\right), w\right\rangle=0 .
\end{aligned}
$$

Observing that

$$
\left|\left(\varepsilon_{1}-\varepsilon_{2}\right)\left\langle-\Delta \partial_{t} u_{1}, w\right\rangle\right| \leq \frac{\left(\varepsilon_{1}-\varepsilon_{2}\right)^{2}}{2}\left\|\nabla \partial_{t} u_{1}\right\|^{2}+\frac{1}{2}\|\nabla w\|^{2}
$$

and by (1.3), we have

$$
\left\langle f\left(u_{1}\right)-f\left(u_{2}\right), w\right\rangle \geq-l\|w\|^{2}
$$

for some $l \geq \lambda_{1}$.

Collecting (3.18)-(3.20), we arrive at

$$
\frac{d}{d t}\left(\|w\|^{2}+\varepsilon_{2}\|\nabla w\|^{2}\right) \leq 2 l\left(\|w\|^{2}+\varepsilon_{2}\|\nabla w\|^{2}\right)+\left(\varepsilon_{1}-\varepsilon_{2}\right)^{2}\left\|\nabla \partial_{t} u_{1}\right\|^{2} .
$$

Thus, by Gronwall's inequality and noting that $\lambda_{1}\|u\|^{2} \leq\|\nabla u\|^{2}$, we have

$$
\|w(t)\|^{2}+\varepsilon_{2}\|\nabla w(t)\|^{2} \leq C\left(\left\|\nabla\left(u_{1}-u_{2}\right)\right\|^{2}+\left(\varepsilon_{1}-\varepsilon_{2}\right)^{2} \int_{\tau}^{t}\left\|\nabla \partial_{t} u_{1}(s)\right\|^{2} d s\right),
$$

where $C$ depends on $t, \tau$ and $|I|$.

Now we divide the argument into two cases.

Case 1: $\varepsilon_{1} \varepsilon_{2} \neq 0$. Without loss of generality, let $\varepsilon_{1} \geq \varepsilon_{2}>0$, from Lemma 3.1 and (3.21), we readily get

$$
\begin{aligned}
& \sup _{g \in \Sigma}\left\|\nabla\left(U_{g}^{\varepsilon_{1}}(t, \tau) u_{1}-U_{g}^{\varepsilon_{2}}(t, \tau) u_{2}\right)\right\|^{2} \\
& \quad \leq C\left(\left\|\nabla\left(u_{1}-u_{2}\right)\right\|^{2}+2\left(\varepsilon_{1}-\varepsilon_{2}\right) \cdot \varepsilon_{1} \int_{\tau}^{t}\left\|\nabla \partial_{t} u_{1}(s)\right\|^{2} d s\right) \\
& \quad \leq Q\left(\left\|\nabla\left(u_{1}-u_{2}\right)\right\|^{2}+\left(\varepsilon_{1}-\varepsilon_{2}\right)\right),
\end{aligned}
$$

where $Q$ depends on $t, \tau,\|g\|_{L_{b}^{2}},\left\|\partial_{t} g\right\|_{L_{b}^{2}}$ and $H_{0}^{1}$-bounds of $B$.

Case 2: $\varepsilon_{1} \varepsilon_{2}=0$. Without loss of generality, let $\varepsilon_{2}=0$, then (3.21) can be simplified as

$$
\|w(t)\|^{2} \leq C\left(\left\|\nabla\left(u_{1}-u_{2}\right)\right\|^{2}+\varepsilon_{1}^{2} \int_{\tau}^{t}\left\|\nabla \partial_{t} u_{1}(s)\right\|^{2} d s\right) .
$$


Multiplying (3.17) by $w$ and using (3.20), we obtain

$$
\|\nabla w(t)\|^{2} \leq C\left(\left\|\partial_{t} w(t)\right\|\|w(t)\|+\varepsilon_{1}^{2}\left\|\nabla \partial_{t} u_{1}(t)\right\|^{2}+\|w(t)\|^{2}\right) .
$$

Hence, on account of Lemmas 3.1, 3.2 and (3.23), similar to (3.22), we find

$$
\sup _{g \in \Sigma}\left\|\nabla\left(U_{g}^{\varepsilon_{1}}(t, \tau) u_{1}-U_{g}^{\varepsilon_{2}}(t, \tau) u_{2}\right)\right\|^{2} \leq Q\left(\left\|\nabla\left(u_{1}-u_{2}\right)\right\|^{2}+\varepsilon_{1}\right) .
$$

Combining (3.22) and (3.25), we can get the expected result.

Proof of Theorem 1.2 If (1.9) is not correct, we can find $\delta>0, \varepsilon_{0} \geq 0$ and $\left\{\varepsilon_{n}\right\}_{n \in \mathbb{N}} \subset[0, \infty)$ with $\varepsilon_{n} \rightarrow \varepsilon_{0}$ such that

$$
\operatorname{dist}_{H_{0}^{1}}\left(\mathcal{A}_{\Sigma}^{\varepsilon_{n}}, \mathcal{A}_{\Sigma}^{\varepsilon_{0}}\right) \geq \delta, \quad \forall n \in \mathbb{N} .
$$

Hence, there exists $\left\{y_{n}\right\}_{n \in \mathbb{N}} \subset \mathcal{A}_{\Sigma}^{\varepsilon_{n}}$ such that

$$
\operatorname{dist}_{H_{0}^{1}}\left(y_{n}, \mathcal{A}_{\Sigma}^{\varepsilon_{0}}\right) \geq \delta, \quad \forall n \in \mathbb{N} .
$$

Let $\mathcal{B}$ be the uniformly (w.r.t. $\sigma \in \Sigma, \varepsilon \in[0, \infty)$ ) absorbing set given by Corollary 3.1. Then we can choose $m>0$ sufficiently large to guarantee that

$$
\bigcup_{\varepsilon \geq 0} \bigcup_{g \in \Sigma} U_{g}^{\varepsilon}(t, 0) \mathcal{B} \subset \mathcal{B}, \quad \forall t \geq m
$$

and

$$
\sup _{g \in \Sigma} \operatorname{dist}\left(U_{g}^{\varepsilon_{0}}(m, 0) \mathcal{B}, \mathcal{A}_{\Sigma}^{\varepsilon_{0}}\right) \leq \frac{\delta}{4}
$$

From Theorem 1.1 we know $\mathcal{A}_{\Sigma}^{\varepsilon_{n}}=\omega_{0, \Sigma}^{\varepsilon_{n}}(\mathcal{B})(n \in \mathbb{N})$. Therefore, there exist sequences $\left\{g_{n}\right\}_{n \in \mathbb{N}} \subset \Sigma,\left\{x_{n}\right\}_{n \in \mathbb{N}} \subset \mathcal{B}$ and $\left\{t_{n}\right\}_{n \in \mathbb{N}} \subset \mathbb{R}^{+}$with $t_{n} \rightarrow \infty$, without loss of generality, we let $t_{n} \geq 2 m$ satisfy

$$
\left\|U_{g_{n}}^{\varepsilon_{n}}\left(t_{n}, 0\right) x_{n}-y_{n}\right\|_{H_{0}^{1}} \leq \frac{\delta}{4}, \quad \forall n \in \mathbb{N} .
$$

Let $\widetilde{x}_{n}=U_{g_{n}}^{\varepsilon_{n}}\left(t_{n}-m, 0\right) x_{n}$ and $g_{n}^{\prime}=T\left(t_{n}-m\right) g_{n}$, by (1.6), (1.7), (3.27) and noticing that $t_{n} \geq$ $2 m$, we have

$$
\left\{\tilde{x}_{n}\right\}_{n \in \mathbb{N}} \subset \mathcal{B}
$$

and

$$
U_{g_{n}}^{\varepsilon_{n}}\left(t_{n}, 0\right) x_{n}=U_{g_{n}}^{\varepsilon_{n}}\left(t_{n}, t_{n}-m\right) \widetilde{x}_{n}=U_{g_{n}^{\prime}}^{\varepsilon_{n}}(m, 0) \widetilde{x}_{n}
$$

On the other hand, due to Lemma 3.3, we can choose $N \in \mathbb{N}$ large enough such that

$$
\left\|U_{g_{N}^{\prime}}^{\varepsilon_{N}}(m, 0) \widetilde{x}_{N}-U_{g_{N}^{\prime}}^{\varepsilon_{0}}(m, 0) \widetilde{x}_{N}\right\| \leq \frac{\delta}{4} .
$$


Therefore, from the above analysis we find

$$
\begin{aligned}
& \operatorname{dist}_{H_{0}^{1}}\left(y_{N}, \mathcal{A}_{\Sigma}^{\varepsilon_{0}}\right) \\
& \leq \operatorname{dist}_{H_{0}^{1}}\left(y_{N}, U_{g_{N}^{\prime}}^{\varepsilon_{N}}(m, 0) \widetilde{x}_{N}\right) \\
& \quad+\operatorname{dist}_{H_{0}^{1}}\left(U_{g_{N}^{\prime}}^{\varepsilon_{N}}(m, 0) \widetilde{x}_{N}, U_{g_{N}^{\prime}}^{\varepsilon_{0}}(m, 0) \widetilde{x}_{N}\right) \\
& \quad+\operatorname{dist}_{H_{0}^{1}}\left(U_{g_{N}^{\prime}}^{\varepsilon_{0}}(m, 0) \widetilde{x}_{N}, U_{g_{N}^{\prime}}^{\varepsilon_{0}}(m, 0) \mathcal{B}\right) \\
& \quad+\operatorname{dist}_{H_{0}^{1}}\left(U_{g_{N}^{\prime}}^{\varepsilon_{0}}(m, 0) \mathcal{B}, \mathcal{A}_{\Sigma}^{\varepsilon_{0}}\right) \\
& \leq \frac{\delta}{4}+\frac{\delta}{4}+0+\frac{\delta}{4}=\frac{3 \delta}{4},
\end{aligned}
$$

which contradicts (3.26).

Next, we prove (1.10). If it is not correct, we can find $\delta>0, t_{0} \in \mathbb{R}, g \in \Sigma, \varepsilon_{0} \geq 0$ and $\left\{\varepsilon_{n}\right\}_{n \in \mathbb{N}} \subset[0, \infty)$ with $\varepsilon_{n} \rightarrow \varepsilon_{0}$ such that

$$
\operatorname{dist}_{H_{0}^{1}}\left(\mathcal{K}_{g}^{\varepsilon_{n}}\left(t_{0}\right), \mathcal{K}_{g}^{\varepsilon_{0}}\left(t_{0}\right)\right) \geq \delta, \quad \forall n \in \mathbb{N}
$$

Let $\mathcal{K}_{g}^{\varepsilon}$ be the kernel of the process $U_{g}^{\varepsilon}(t, \tau)$. By (3.28), for every $n \in \mathbb{N}$, there exists a complete trajectory $u_{n}(\cdot) \in \mathcal{K}_{g}^{\varepsilon_{n}}$ satisfying

$$
\operatorname{dist}_{H_{0}^{1}}\left(u_{n}\left(t_{0}\right), \mathcal{K}_{g}^{\varepsilon_{0}}\left(t_{0}\right)\right) \geq \delta, \quad \forall n \in \mathbb{N},
$$

and

$$
u_{n}(t)=U_{g}^{\varepsilon_{n}}(t, \tau) u_{n}(\tau), \quad \forall t \geq \tau, \forall \tau \in \mathbb{R}
$$

For every $s \in \mathbb{R}$, since $u_{n}(s) \in \mathcal{K}_{g}^{\varepsilon_{n}}(s) \subset \mathcal{A}_{\Sigma}^{\varepsilon_{n}}$, by (1.8), (1.9) and the compactness of $\mathcal{A}_{\Sigma}^{\varepsilon_{0}}$, there exists $u(s) \in \mathcal{A}_{\Sigma}^{\varepsilon_{0}}$ such that

$$
\left\|u_{n}(s)-u(s)\right\|_{H_{0}^{1}} \stackrel{n \rightarrow \infty}{\longrightarrow} 0 .
$$

Consequently, Lemma 3.3 yields

$$
\left\|U_{g}^{\varepsilon_{n}}(t, \tau) u_{n}(\tau)-U_{g}^{\varepsilon_{0}}(t, \tau) u(\tau)\right\|_{H_{0}^{1}} \stackrel{n \rightarrow \infty}{\longrightarrow} 0, \quad \forall t \geq \tau, \forall \tau \in \mathbb{R} .
$$

Combining (3.31) and (3.32), taking $n \rightarrow \infty$ in (3.30), we find

$$
u(t)=U_{g}^{\varepsilon_{0}}(t, \tau) u(\tau), \quad \forall t \geq \tau, \forall \tau \in \mathbb{R}
$$

that is, $u(\cdot) \in \mathcal{K}_{g}^{\varepsilon_{0}}$ and $u(t) \in \mathcal{K}_{g}^{\varepsilon_{0}}(t)(\forall t \in \mathbb{R})$.

Hence

$$
\operatorname{dist}_{H_{0}^{1}}\left(u_{n}\left(t_{0}\right), \mathcal{K}_{g}^{\varepsilon_{0}}\left(t_{0}\right)\right) \leq \operatorname{dist}_{H_{0}^{1}}\left(u_{n}\left(t_{0}\right), u\left(t_{0}\right)\right) \stackrel{n \rightarrow \infty}{\longrightarrow} 0,
$$

and this contradicts (3.29). The proof is completed. 


\section{Acknowledgements}

We would like to thank the referee for invaluable comments and insightful suggestions.

\section{Funding}

The first author was supported by the National Natural Science Foundation of China (Grant No. 11501096) and the Fundamental Research Funds for the Central Universities (Grant No. 2232015D3-36). The third author was supported by the National Natural Science Foundation of China (Grant No. 11671075).

\section{Competing interests}

The authors declare that they have no competing interests.

\section{Authors' contributions}

All authors have made the same contribution and finalized the current version of this manuscript.

\section{Publisher's Note}

Springer Nature remains neutral with regard to jurisdictional claims in published maps and institutional affiliations.

Received: 6 March 2017 Accepted: 19 May 2017 Published online: 06 June 2017

\section{References}

1. Aifantis, E: On the problem of diffusion in solids. Acta Mech. 37, 265-296 (1980)

2. Truesdell, C, Noll, W: The Nonlinear Field Theories of Mechanics. Springer, Berlin (1995)

3. Anh, C, Bao, T: Dynamics of non-autonomous nonclassical diffusion equation on $\mathbb{R}^{n}$. Commun. Pure Appl. Anal. 11 1231-1252 (2012)

4. Anh, C, Bao, T: Pullback attractors for a class of non-autonomous nonclassical diffusion equations. Nonlinear Anal. 73, 399-412 (2010)

5. Conti, M, Marchini, E, Pata, V: Nonclassical diffusion with memory. Math. Methods Appl. Sci. 38, 948-958 (2015)

6. Rivero, F: Time dependent perturbation in a non-autonomous non-classical parabolic equation. Discrete Contin. Dyn. Syst. 18, 209-221 (2013)

7. Sun, C, Wang, S, Zhong, C: Global attractors for a nonclassical diffusion equation. Acta Math. Sin. 23, 1271-1280 (2007)

8. Xiao, Y: Attractors for a nonclassical diffusion equation. Acta Math. Sin. 18, 273-276 (2002)

9. Sun, C, Yang, M: Dynamics of the nonclassical diffusion equations. Asymptot. Anal. 59, 51-81 (2008)

10. Wang, S, Li, D, Zhong, C: On the dynamics of a class of nonclassical parabolic equations. J. Math. Anal. Appl. 317 565-582 (2006)

11. Wang, X, Yang, L, Zhong, C: Attractors for the nonclassical diffusion equation with fading memory. J. Math. Anal. Appl. $362,327-337(2010)$

12. Wang, $X$, Yang, L: Attractors for the non-autonomous nonclassical diffusion equation with fading memory. Nonlinear Anal. 71, 5733-5746 (2009)

13. Wang, L, Wang, Y, Qin, Y: Upper semicontinuity of attractors for nonclassical diffusion equations in $H^{\prime}\left(\mathbb{R}^{3}\right)$. Appl. Math. Comput. 240, 51-61 (2014)

14. Chepyzhov, V, Vishik, M: Attractors for Equations of Mathematical Physics. American Mathematical Society Colloquium Publications, vol. 49. American Mathematical Society, Providence (2002)

15. Lu, S: Attractors for nonautonomous reaction-diffusion systems with symbols without strong translation compactness. Asymptot. Anal. 54, 197-210 (2007)

16. Song, $\mathrm{H}, \mathrm{Ma}$, S, Zhong, C: Attractors of non-autonomous reaction-diffusion equations. Nonlinearity 22, 667-681 (2009)

17. Xie, Y, Zhu, K, Sun, C: The existence of uniform attractors for non-autonomous reaction-diffusion equations on the whole space. J. Math. Phys. 53, 082703 (2012)

18. Zelik, S: Strong uniform attractors for non-autonomous dissipative PDEs with non translation-compact external forces. Discrete Contin. Dyn. Syst. 20, 781-810 (2015)

19. Chepyzhov, V, Vishik, M: Attractors of nonautonomous dynamical systems and their dimension. J. Math. Pures Appl. 73, 279-333 (1994)

20. Moise, I, Rosa, R, Wang, X: Attractors for noncompact nonautonomous systems via energy equations. Discrete Contin. Dyn. Syst. 10, 473-496 (2004)

21. Lu, S, Wu, H, Zhong, C: Attractors for nonautonomous 2D Navier-Stokes equations with normal external forces. Discrete Contin. Dyn. Syst. 13, 701-719 (2005)

22. Chen, G, Zhong, C: Uniform attractors for non-autonomous p-Laplacian equations. Nonlinear Anal. 68, 3349-3363 (2008) 